\title{
Excitonic properties of polar, semipolar, and nonpolar InGaN/GaN strained quantum wells with potential fluctuations
}

\author{
$\operatorname{AUTHOR}(\mathrm{S}):$ \\ Funato, M.; Kawakami, Y.
}

\section{CITATION:}

Funato, M....[et al]. Excitonic properties of polar, semipolar, and nonpolar InGaN/GaN strained quantum wells with potential fluctuations. JOURNAL OF APPLIED PHYSICS 2008, 103(9): 093501.

ISSUE DATE:

2008-05-01

URL:

http://hdl.handle.net/2433/84583

\section{RIGHT:}

Copyright 2008 American Institute of Physics. This article may be downloaded for personal use only. Any other use requires prior permission of the author and the American Institute of Physics. 


\title{
Excitonic properties of polar, semipolar, and nonpolar InGaN/GaN strained quantum wells with potential fluctuations
}

\author{
M. Funato ${ }^{\text {a) }}$ and Y. Kawakami \\ Department of Electronic Science and Engineering, Kyoto University, Kyoto 615-8510, Japan
}

(Received 2 November 2007; accepted 6 February 2008; published online 1 May 2008)

\begin{abstract}
Excitonic properties of polar, semipolar, and nonpolar InGaN/GaN strained quantum wells (QWs) were investigated in terms of exciton localization and polarization-induced electric fields. The spontaneous emission lifetimes measured at $\sim 10 \mathrm{~K}$ for the (0001) polar QWs were $1.4 \mathrm{~ns}$ at an emission wavelength of $400 \mathrm{~nm}$, but increased monotonically to $85 \mathrm{~ns}$ at $520 \mathrm{~nm}$. On the other hand, those for $\{11 \overline{2} 2\}$ and $\{1 \overline{1} 01\}$ semipolar QWs and $\{11 \overline{2} 0\}$ and $\{1 \overline{1} 00\}$ nonpolar QWs were on the order of a few hundred picoseconds and independent of the emission wavelength. To quantitatively discuss these results, the crystalline orientation dependence of the spontaneous emission lifetime of $1 s$ heavy hole excitons in $\mathrm{InGaN} / \mathrm{GaN}$ QWs at $0 \mathrm{~K}$ was calculated, when lateral confinements were considered to express well-reported potential fluctuations. It is revealed that both the crystalline orientation and lateral confinement vary the spontaneous emission lifetime by orders of magnitude. Analyses of the experimental results suggest that excitons in the (0001) polar QWs are more strongly localized as the In composition increases, but the semipolar and nonpolar QWs exhibit the opposite tendency. These tendencies are attributed to differences in the growth characteristics. (0) 2008 American Institute of Physics. [DOI: 10.1063/1.2903592]
\end{abstract}

\section{INTRODUCTION}

Light emitters based on nitride semiconductors consist of quantum wells ${ }^{1}(\mathrm{QWs})$ and show interesting phenomena due to strain induced by the lattice mismatch between the constituent materials. For example, a large lattice mismatch of $11 \%$ between $\mathrm{GaN}$ and $\mathrm{InN}$ makes it difficult to grow uniformly mixed $\mathrm{InGaN}_{\text {alloys }}{ }^{2}$ and, consequently, causes compositional fluctuations. It has been reported that such fluctuations form nanometer-scale potential minima in InGaN QWs, which work as carrier localization centers with high internal quantum efficiencies (IQEs) ${ }^{3-5}$ Furthermore, nitride QWs are usually $c$ oriented, and inevitably experience strain-induced piezoelectric polarizations. This piezoelectric polarization along with the spontaneous polarization induces electric fields in the QWs, which lower the optical transition probability. ${ }^{6,7}$ Therefore, to achieve higher IQEs, it is important to reduce the electric field. References 8 and 9 calculate the variation of the polarization field as a function of tilt of the $c$ axis and have demonstrated that the polarization field in strained $\mathrm{InGaN}$ on $\mathrm{GaN}$ becomes zero near $39^{\circ}-55^{\circ}$ and $90^{\circ}$. (The scattering of the calculated semipolar direction to provide a zero electric field is due to ambiguity of the material parameters.) These calculations suggest that semipolar and nonpolar QWs are promising for higher IQEs.

Experimentally, we have demonstrated that semipolar $\{11 \overline{2} 2\}$ QWs, which form an angle of $58^{\circ}$ with respect to (0001), can be fabricated by a regrowth technique ${ }^{10-12}$ or homoepitaxy, ${ }^{13,14}$ and that GaN grown on GaAs $\{114\} \mathrm{B}$ substrates tilts $\sim 30^{\circ} .{ }^{15}$ In particular, the $\{11 \overline{2} 2\}$ QWs involve electric fields much weaker than those in the (0001) polar QWs. On the other hand, the growth on nonpolar planes such

${ }^{a)}$ Electronic mail: funato@kuee.kyoto-u.ac.jp. as $\{1 \overline{1} 00\}$ ( $M$ plane) (Ref. 16) and $\{11 \overline{2} 0\}$ (A plane), ${ }^{17-19}$ which form $90^{\circ}$ with respect to (0001), has also been performed. It has been demonstrated that $\{1 \overline{1} 00\} \mathrm{GaN} / \mathrm{AlGaN}$ multiple QWs grown on $\gamma-\mathrm{LiAlO}_{2}(100)$ substrates possess a higher IQE compared to (0001) polar QWs. ${ }^{16}$

In this study, we investigate the optical properties of polar, semipolar, and nonpolar InGaN/GaN QWs in terms of exciton localization and internal electric fields. The spontaneous emission lifetime, which is sensitive to both the factors, was initially measured for (0001) polar QWs, $\{11 \overline{2} 2\}$ and $\{1 \overline{1} 01\}$ semipolar QWs, and $\{11 \overline{2} 0\}$ and $\{1 \overline{1} 00\}$ nonpolar QWs. Next, theoretical considerations were given to the orientation dependence of the excitonic properties such as the spontaneous emission lifetime and binding energy, where to express localization states, quantum disks (QDs) were assumed. The effect of carrier injection was also examined because the screening of the electric fields by injected carriers affects the optical properties. ${ }^{20-23}$ Finally, the experimental results were analyzed with the theoretical considerations to evaluate the localization degree of excitons in those QWs.

\section{LIFETIME MEASUREMENTS}

Time resolved (TR) photoluminescence (PL) measurements were performed for polar, semipolar, and nonpolar $\mathrm{InGaN} / \mathrm{GaN}$ QWs at $\sim 10 \mathrm{~K}$.

The QWs were grown by metalorganic vapor phase epitaxy on sapphire (0001) substrates. The polar (0001) QWs were conventional planar QWs, while the semipolar $\{11 \overline{2} 2\}$ and $\{1 \overline{1} 01\}$ QWs and the nonpolar $\{11 \overline{2} 0\}$ and $\{1 \overline{1} 00\}$ QWs were fabricated on GaN facet structures created through regrowth. The growth procedure for the semipolar and nonpolar facet QWs was detailed in Refs. 10-12. All the QWs had 


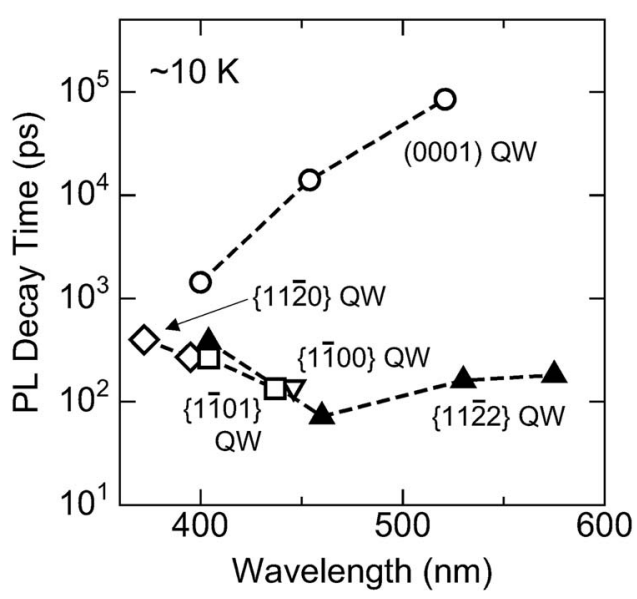

FIG. 1. PL lifetimes of (0001), $\{11 \overline{2} 2\},\{1 \overline{1} 01\},\{11 \overline{2} 0\}$, and $\{1 \overline{1} 00\}$ InGaN/GaN QWs measured at $\sim 10 \mathrm{~K}$.

InGaN well widths of $2-3 \mathrm{~nm}$, which were measured by transmission electron microscopy and/or x-ray diffraction.

The excitation pulses for TRPL were from a frequency doubled $\mathrm{Ti}: \mathrm{Al}_{2} \mathrm{O}_{3}$ laser. The pulse repetition rate and width were $80 \mathrm{MHz}$ and $1.5 \mathrm{ps}$, respectively. The output wavelength was set to selectively excite the InGaN wells and ranged from 355 to $380 \mathrm{~nm}$. The excitation energy density was between 0.006 and $1.3 \mu \mathrm{J} / \mathrm{cm}^{2}$. From those quantities, the photogenerated carrier density was estimated to be, at most, $2 \times 10^{17} \mathrm{~cm}^{-3}$. Thus, the current experimental condition can be regarded as a weak excitation condition because such a carrier density barely affects internal electric fields, as discussed below. The PL was detected by a streak camera. The measured PL lifetime corresponds to the radiative recombination lifetime because a temperature of $\sim 10 \mathrm{~K}$ has negligible nonradiative processes.

Figure 1 depicts the results. The semipolar and nonpolar QWs always displayed faster lifetimes than the (0001) QWs. Longer wavelengths enlarged these differences. It is noteworthy that, in the blue-green to red spectral range, which is where the current polar QWs suffer from low IQEs primarily due to the polarization effect, the lifetimes in the semipolar and nonpolar QWs were shortened by more than two orders of magnitude, suggesting better IQEs. Figure 1 also demonstrates that the lifetimes in the semipolar and nonpolar QWs are on the same curve, although the strength of the internal electric field may differ in these QWs. In order to quantitatively analyze these observations, theoretical considerations are given in the following sections.

\section{THEORETICAL CONSIDERATIONS}

\section{A. Calculation procedures}

InGaN/GaN QWs with lateral confinement (due to potential fluctuations) were used as model structures for the calculation along with the following assumptions: (i) the GaN barrier thickness was infinite, (ii) $\mathrm{GaN}$ was completely relaxed, and (iii) $\mathrm{InGaN}$ was coherently grown on $\mathrm{GaN}$. The assumed temperature was $0 \mathrm{~K}$, so that a thermalization effect was not considered. Table I lists the parameters used, but the parameters of $\mathrm{GaN}$ were substituted for those of $\mathrm{InN}$ if the
InN parameters were unavailable (N/A). The dependences of the dielectric constant and the effective mass on the crystalline orientation were simply obtained by interpolation.

The calculation was performed for the QDs with a tilt of $0^{\circ}$ ( $c$ oriented), $30^{\circ}, 60^{\circ}$, or $90^{\circ}$. These angles were chosen to reproduce realistic examples introduced in Sec. I. First, the strength of the electric field along the surface normal in the $\mathrm{InGaN} / \mathrm{GaN}$ heterostructures was calculated. The calculation is essentially the same as that proposed in Ref. 9, but the spontaneous polarization is considered. The results are shown in Fig. 2, which demonstrates that $c$-oriented InGaN involves the strongest internal electric field, followed by InGaN tilted $30^{\circ}, 60^{\circ}$, and then $90^{\circ}$. For example, the electric fields in $\mathrm{In}_{0.20} \mathrm{Ga}_{0.80} \mathrm{~N}$ on $\mathrm{GaN}$ were 2.1, 0.85, -0.55 , and $0 \mathrm{MV} / \mathrm{cm}$ for $0^{\circ}, 30^{\circ}, 60^{\circ}$, and $90^{\circ}$, respectively. Next, the band gap under the compressive strain induced by the lattice mismatch was calculated. ${ }^{31}$ Using the obtained strength of the electric field and band gap, the band lineup of the QW was determined. ${ }^{36}$ At this stage, lateral confinement has yet to be included. Hence, the one-dimensional Schrödinger equation was numerically solved. When the electric field was screened by free carriers, the Schrödinger and Poisson equations were solved self-consistently. Finally, the spontaneous emission lifetime and binding energy of $1 s$ excitons in QDs were calculated by modifying the method proposed in Ref. 37 so that the QD possessed a cylindrical shape, and the vertical and lateral confinements were described by the above-obtained QW potential profile and harmonicoscillator-type parabolic potential, respectively.

It has often been reported that the PL lifetimes of InGaN QWs at a low temperature decrease in the short-wavelength tail of an emission band as the wavelength decreases, ${ }^{5}$ which is well accounted for by the weakly localized exciton model. ${ }^{38}$ However, the structures of the localization centers are controversial, and therefore, we adopted a rather simple model for the current calculation. In addition, the weak localization suggests that the In composition in $\operatorname{In}_{x} \mathrm{Ga}_{1-x} \mathrm{~N}$ emission centers does not differ significantly from that in surrounding $\operatorname{In}_{y} \mathrm{Ga}_{1-y} \mathrm{~N}(y<x)$, which results in weak inplane electric fields. This is why the in-plane electric fields were neglected.

To confirm the validity of the current calculation procedures and the model, the calculated results were compared to experimental data, as shown in Fig. 3. Experimentally, a (0001) InGaN/GaN single QW emitting at $490 \mathrm{~nm}$ at $15 \mathrm{~K}$ was characterized. The designed well width and In composition were $3 \mathrm{~nm}$ and 23\%, respectively. Scanning near field optical microscopy (SNOM) with a spatial resolution of $30 \mathrm{~nm}$ showed a few spiky luminescent peaks in each spectrum, ${ }^{39}$ indicating that there are a few luminescence centers within the $30 \mathrm{~nm}$ aperture. This result suggests that the dimensions of luminescence centers are a few nanometers. On the other hand, TRPL spectroscopy was also conducted for the same sample at $15 \mathrm{~K}$ under a weak excitation $\left(300 \mathrm{~nJ} / \mathrm{cm}^{2}\right)$. The dependence of the PL decay on the emission wavelength could be well described by the weakly localized exciton model, which gave a radiative recombination lifetime of 100-200 ns and a characteristic energy of $35 \mathrm{meV}$. ${ }^{40}$ These experimental results are shown as the dot- 
TABLE I. Physical parameters used for the calculation.

\begin{tabular}{|c|c|c|}
\hline & $\mathrm{GaN}$ & $\mathrm{InN}$ \\
\hline Lattice parameter $(\mathrm{nm})$ & $a=0.3189, c=0.5185$ & $a=0.3536, c=0.5701^{\mathrm{a}}$ \\
\hline Piezoelectric constant $\left(\mathrm{C} / \mathrm{m}^{2}\right)$ & $e_{33}=0.63, e_{31}=e_{15}=-0.32^{\mathrm{b}}$ & $e_{33}=0.43, e_{31}=e_{15}=-0.22^{\mathrm{c}}$ \\
\hline Spontaneous polarization $\left(\mathrm{C} / \mathrm{m}^{2}\right)^{\mathrm{d}}$ & -0.029 & -0.032 \\
\hline Elastic constant $(\mathrm{GPa})$ & $\begin{array}{c}C_{11}=365, C_{12}=135, \\
C_{13}=114, C_{33}=381, C_{44}=109^{\mathrm{e}}\end{array}$ & $\begin{array}{c}C_{11}=223, C_{12}=115, \\
C_{13}=92, C_{33}=224, C_{44}=48^{\mathrm{f}}\end{array}$ \\
\hline Dielectric constant & & \\
\hline (for electric fields) & $10.4(c \|), 9.5(c \perp)^{\mathrm{g}}$ & $15.3^{\text {h }}$ \\
\hline (for excitons) & $8.57(c \|), 7.87(c \perp)^{\mathrm{i}}$ & $15.3^{\mathrm{h}}$ \\
\hline Effective masses $\left(/ m_{0}\right)^{\mathrm{j}}$ & $\begin{aligned} m_{e(c \|)} & =0.19, m_{e(c \perp)}=0.17, \\
m_{\mathrm{HH}(c \|))} & =1.76, m_{\mathrm{HH}(c \perp)}=1.69,\end{aligned}$ & $\begin{aligned} m_{e(c \| l)} & =0.11, m_{e(c \perp)}=0.10, \\
m_{\mathrm{HH}(c \|)} & =1.56, m_{\mathrm{HH}(c \perp)}=1.68,\end{aligned}$ \\
\hline Energy gap (eV) & 3.50 & $0.69^{\mathrm{k}}$ \\
\hline Bowing parameter $(\mathrm{eV})$ & \multicolumn{2}{|c|}{$2.3^{1}$} \\
\hline \multicolumn{3}{|l|}{ Deformation potentials $(\mathrm{eV})$} \\
\hline$\Xi_{\mathrm{D}}-\left\{D_{1}-\left(C_{33} / C_{13}\right) D_{2}\right\}^{\mathrm{m}}$ & $38.9^{\mathrm{n}}$ & N/A \\
\hline$D_{3}-\left(C_{33} / C_{13}\right) D_{4}^{\mathrm{m}}$ & $23.6^{\mathrm{n}}$ & N/A \\
\hline Crystal field parameter $(\mathrm{eV})^{\mathrm{o}}$ & 0.042 & 0.041 \\
\hline Spin-orbit parameter $(\mathrm{eV})^{\circ}$ & 0.013 & 0.001 \\
\hline Valence band offset $(\mathrm{eV})^{\mathrm{o}}$ & \multicolumn{2}{|c|}{0.48} \\
\hline
\end{tabular}

${ }^{\mathrm{a}}$ Reference 24.

${ }^{\mathrm{b}}$ Reference 25.

${ }^{\mathrm{c}}$ Reference 1.

${ }^{\mathrm{d}}$ Reference 26.

${ }^{\mathrm{e}}$ Reference 27.

${ }^{\mathrm{f}}$ Reference 28.

${ }^{\mathrm{g}}$ Reference 29 .

${ }^{\text {h }}$ Reference 30.

${ }^{\mathrm{i}}$ Reference 31.

${ }^{\mathrm{j}}$ Reference 32.

${ }^{\mathrm{k}}$ Reference 33 .

${ }^{\mathrm{l}}$ Reference 34.

${ }^{\mathrm{m}}$ The notations are the same as those in Ref. 31 .

${ }^{\mathrm{n}}$ Reference 31.

${ }^{\circ}$ Reference 35 .

ted region in Fig. 3. Theoretically, the spontaneous emission lifetime in $c$-oriented InGaN/GaN QDs was calculated for various degrees of lateral confinement. The well width and In composition were chosen to maintain the transition energy in QDs with a radius $(R)$ of a few nanometers at $2.53 \mathrm{eV}$ $(=490 \mathrm{~nm}$ in wavelength). The curves in Fig. 3 are the obtained results. For QDs with a radius larger than approxi-

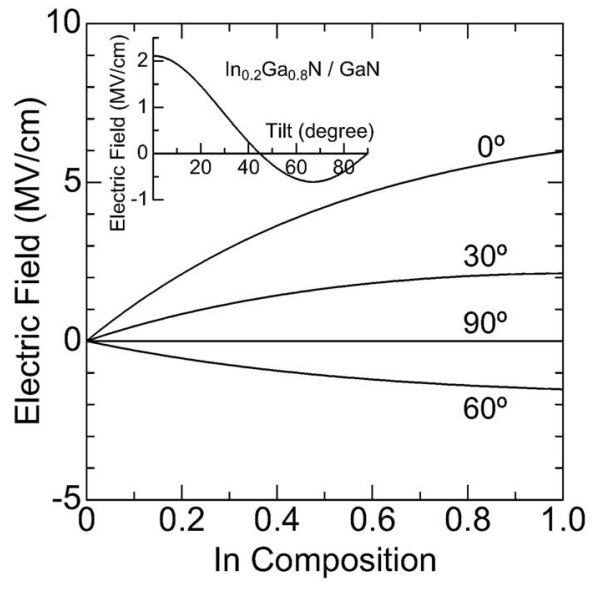

FIG. 2. Calculated electric fields along the surface normal due to piezoelectric and spontaneous polarizations as a function of In composition. Tilt of the $c$ axis is a parameter. The inset shows the dependence of the electric field on the tilt when the In composition is $20 \%$. mately $60 \mathrm{~nm}$, the lifetimes were constant and corresponded to the QW lifetimes. As the radius decreased, the lifetime became longer because a smaller radius caused the wave vector of excitons to spread in the $\boldsymbol{k}$ space, which reduced the number of excitons contributing to radiative recombination. We would like to emphasize that the theoretical curves go through the experimentally determined area when a well

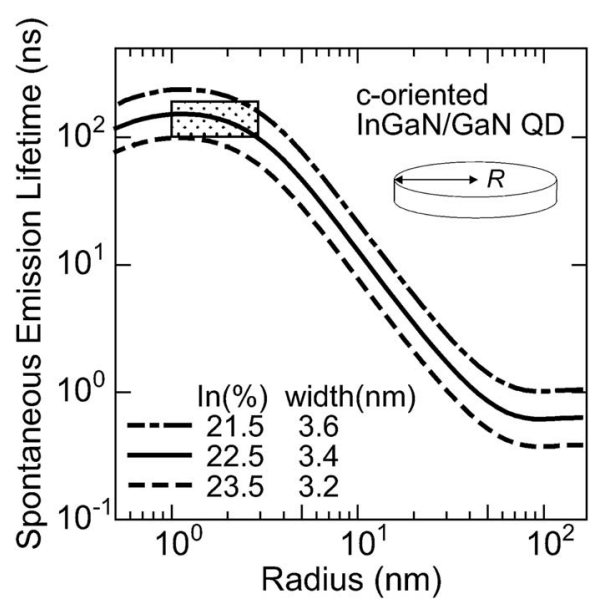

FIG. 3. Calculated variation of the spontaneous emission lifetime in the $c$-oriented $\mathrm{InGaN} / \mathrm{GaN}$ QDs as a function of QD radius $(R)$. Dotted region designates the experimental results of TRPL and SNOM. 


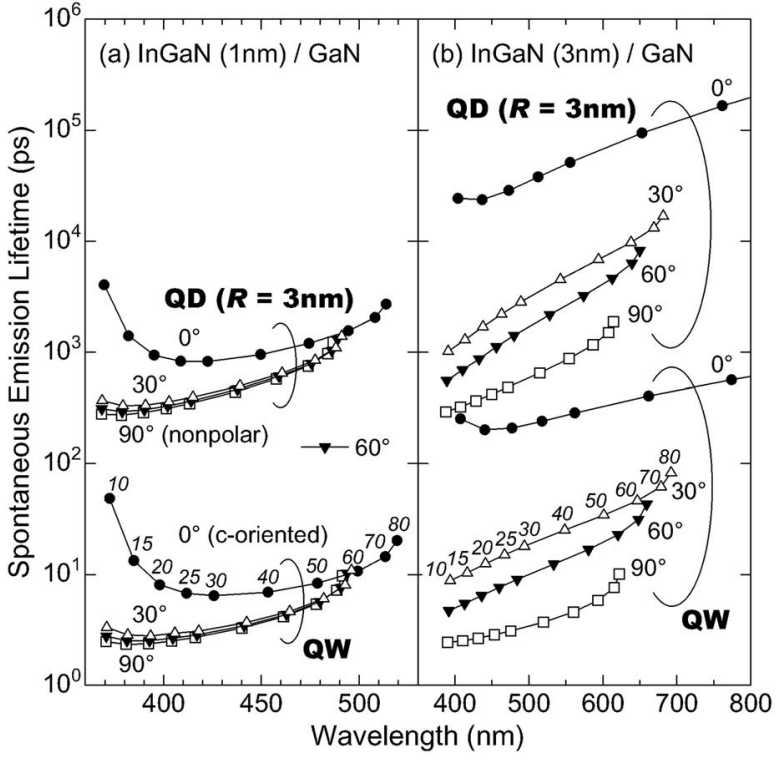

FIG. 4. Calculated spontaneous emission lifetimes in (a) 1-nm-thick and (b) 3-nm-thick InGaN/GaN QWs and QDs with $R=3 \mathrm{~nm}$. In composition is $10 \%, 15 \%, 20 \%, 25 \%, 30 \%, 40 \%, 50 \%, 60 \%, 70 \%$, and $80 \%$ from left to right for all the QWs and QDs, as shown representatively for the QWs by the numbers in italics.

width of $3.4 \pm 0.2 \mathrm{~nm}$ and an In composition of $22.5 \mp 1.0 \%$, which agree well with the design, were assumed. Because a characteristic energy of $\sim 35 \mathrm{meV}$, which was derived from the weakly localization model, can be realized by a small fluctuation in the In composition and/or thickness of $<10 \%$, the result shown in Fig. 3 validates the calculation in this study.

\section{B. Spontaneous emission lifetime}

Figure 4 compares the spontaneous emission lifetimes of QWs and QDs with a tilt of $0^{\circ}, 30^{\circ}, 60^{\circ}$, or $90^{\circ}$. The InGaN thickness was (a) $1 \mathrm{~nm}$ or (b) $3 \mathrm{~nm}$ and the radius $(R)$ for the lateral confinement was $3 \mathrm{~nm}$. The horizontal axis (wavelength) corresponds to the transition energy determined by the In composition in the InGaN wells. The In composition increases from left to right as $10 \%, 15 \%, 20 \%, 25 \%, 30 \%$, $40 \%, 50 \%, 60 \%, 70 \%$, and $80 \%$ for all the QWs and QDs. Figure 4 clearly shows that through almost the entire wavelength range, i.e., for almost all the In compositions, the lifetime became longer in the order $0^{\circ}>30^{\circ}>60^{\circ}>90^{\circ}$. This order agrees with that of the strength of the internal electric field revealed in Sec. III A. Thus, Fig. 4 quantitatively clarifies how much the electric field slows the radiative recombination process.

\section{1-nm-thick $Q W s$ and $Q D s$}

Let us discuss the results for 1-nm-thick InGaN/GaN QWs without a lateral confinement. The semipolar QWs tilted $30^{\circ}$ and $60^{\circ}$ showed properties nearly identical to those of the nonpolar QWs, indicating that the influence of the electric field in 1-nm-thick QWs is avoidable with those tilts, and that the optical transition probabilities of those semipolar and nonpolar QWs are equivalent.
At a wavelength around $500 \mathrm{~nm}$, the QWs with stronger internal electric fields exhibited shorter lifetimes, which at first glance, is inconsistent. To understand this property, the In composition must be considered. At a certain wavelength, the In composition in QWs with weaker electric fields becomes higher due to a smaller quantum confinement Stark effect, which leads to (i) an increased internal electric field and (ii) a lower optical transition probability due to a narrower bulk band gap. Both (i) and (ii) slow the radiative process, which is why tilted QWs exhibit longer lifetimes in the longer wavelength range.

The u shape observed in Fig. 4(a) can be explained as follows. For shorter wavelengths, the carrier confinement is weaker due to lower In compositions. Thus, the carriers experience a lower barrier, which makes it easier to escape from the InGaN well to the $\mathrm{GaN}$ barrier. Because the effective mass of electrons is lighter than that of holes, more electrons penetrate into the barriers, which results in a decreased overlap between the electrons and holes. This trend becomes remarkable in the presence of an internal electric field because the electric field pushes carriers toward the barriers and promotes penetration. Because the spontaneous emission lifetime is inversely proportional to the square overlap of electrons and holes, the penetration of carriers into the barriers gives rise to longer spontaneous emission lifetimes. On the other hand, for longer wavelengths, the In composition in the InGaN well becomes larger, which lowers the optical transition probability by the same mechanism discussed in the previous paragraph.

The effect of lateral confinement was investigated using QDs with the same thickness and $R=3 \mathrm{~nm}$. The calculated results are plotted also in Fig. 4(a). All the emission lifetimes of the QDs became considerably longer relative to those of the QWs. The reason for this has already been described in the discussion with Fig. 3. On the other hand, the relationship among the emission lifetimes of the QDs with the different tilts was well preserved because the internal electric field, which causes the lifetime difference due to the tilts, is perpendicular to the lateral confinement. Therefore, to avoid the influence of the electric field on the optical properties, the electric field itself should be eliminated.

According to our model, the lateral confinement of excitons in QDs with $R=3 \mathrm{~nm}$ produces a factor of 100 increase in the spontaneous emission lifetime, as compared to QWs without lateral confinement. On the other hand, previous experimental results suggest that lateral confinement of excitons by potential fluctuations causes an increase in the IQE. ${ }^{3-5}$ These results can be reconciled by assuming that lateral confinement causes the nonradiative lifetime to increase by an even larger factor than the radiative lifetime, due to suppression of carrier capture by nonradiative centers.

\section{3-nm-thick QWs and QDs}

The spontaneous emission lifetimes calculated for thicker 3-nm-thick QWs and QDs $(R=3 \mathrm{~nm})$ are presented in Fig. 4(b). Let us first consider the effect of QW thickness. Comparing the 3-nm-thick QWs in Fig. 4(b) to the 1-nm-thick QWs in Fig. 4(a) clearly demonstrates that the difference in the lifetimes due to the tilt is more pronounced 


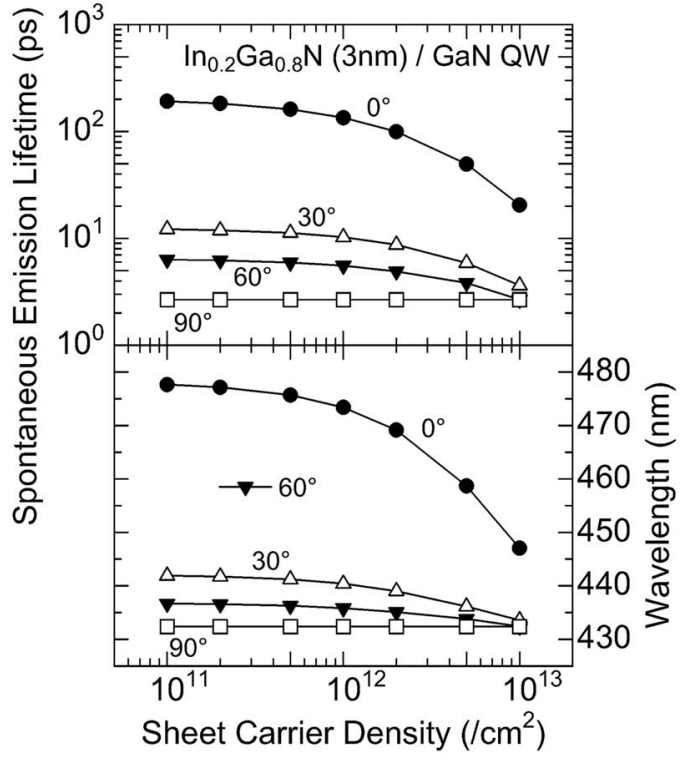

FIG. 5. Variations in the spontaneous emission lifetime and emission peak wavelength in 3-nm-thick $\mathrm{In}_{0.2} \mathrm{Ga}_{0.8} \mathrm{~N} / \mathrm{GaN}$ QWs caused by carrier injection.

in thicker QWs. That is, the contribution of the polarization effect on the exciton recombination process becomes larger as the well width increases. This is consistent with reported experimental results that electric fields affect the optical properties more strongly in thicker QWs. ${ }^{41}$

On the other hand, comparing the 3-nm-thick QWs to the QDs [Fig. 4(b)] confirms that the lateral confinement elongates the lifetime by two orders of magnitude, but barely influences the variation of the lifetimes due to the crystalline tilts. Furthermore, a slight blueshift of $\sim 18 \mathrm{meV}$ is observed for the QDs due to lateral confinement. These properties are quite similar to those in the 1-nm-thick QWs and QDs [Fig. $4(\mathrm{a})]$.

\section{Screening of polarization fields by carrier injection}

Free carriers injected into InGaN wells can screen the polarization fields and improve the optical transition probability. ${ }^{20-23}$ In this section, this effect on the radiative lifetime of $1 s$ excitons is investigated by solving the Schrödinger and Poisson equations self-consistently. The estimated typical sheet carrier density in light emitting diodes (LEDs) was $7 \times 10^{11} \mathrm{~cm}^{-2}$, assuming an operation current of $20 \mathrm{~mA}$, a size of $300 \times 300 \mu \mathrm{m}$, and a recombination lifetime of $5 \mathrm{~ns}$. For laser diodes (LDs), it was $\sim 10^{13} \mathrm{~cm}^{-2}$. Therefore, the sheet carrier density was changed from 1.0 $\times 10^{11}$ to $1.0 \times 10^{13} \mathrm{~cm}^{-2}$.

It was found that the degrees of carrier-induced lifetime variation are rather insensitive to lateral confinement and In$\mathrm{GaN}$ thickness. The former is because the internal electric field is perpendicular to the lateral confinement, while the latter is because the carriers experience only triangular potential profiles formed by the internal electric field, which is independent of the InGaN thickness as long as the In composition is unchanged. Therefore, the results for 3-nm-thick $\mathrm{In}_{0.2} \mathrm{Ga}_{0.8} \mathrm{~N} / \mathrm{GaN}$ QWs are representatively demonstrated. Figure 5 shows variations in the emission lifetime and tran-

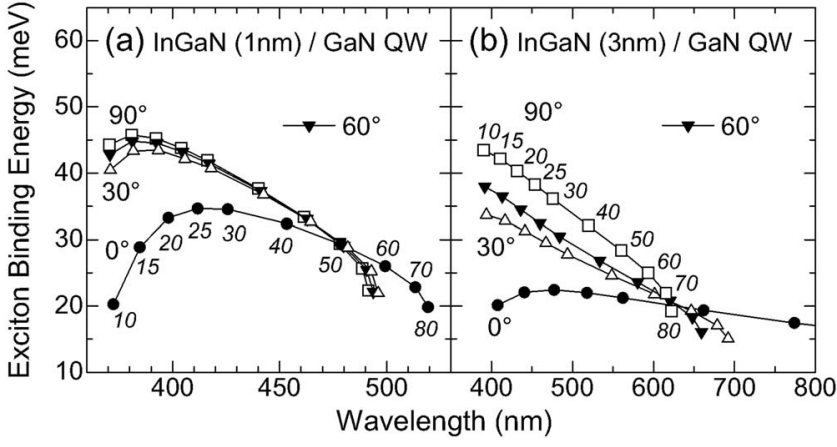

FIG. 6. Binding energies of $1 s$ heavy hole excitons in (a) 1-nm-thick QWs and (b) 3-nm-thick QWs. The numbers in italics indicate the In composition.

sition energy due to the injected carrier density for different tilts. The lifetimes of those QWs were not identical even under a carrier density of $1.0 \times 10^{13} \mathrm{~cm}^{-2}$, which suggests that the electric field remains in LDs under operation. For (0001) polar InGaN/GaN QWs (Ref. 42) and GaN/AlGaN QWs ${ }^{43}$ partial screening under the lasing condition has been documented. The present calculation suggests that the electric field plays a role in the lasing and LED properties of semipolar QWs as well.

The same calculations were performed for polar and semipolar QWs with various In compositions. It is found that increasing the injected carrier density more drastically decreases the lifetime and emission wavelength for QWs with lower and higher In compositions, respectively. Therefore, to assess the internal electric field, it is better to use the lifetime when the In composition is low, but the emission peak shift is more appropriate when the In composition is high.

\section{Binding energy of $1 s$ heavy hole excitons}

The $1 s$ heavy hole exciton binding energy, which is the energy difference between the binding energies with and without consideration of the Coulomb term in the Schrödinger equation, was calculated for both QWs and QDs. The thickness was 1 or $3 \mathrm{~nm}$, and the radius for the lateral confinement was $3 \mathrm{~nm}$. Figure 6 shows the results for the QWs. The binding energies were strengthened in the semipolar and nonpolar QWs, particularly in the shorter wavelength range, and exceeded the RT thermal energy (26 meV) even in QWs.

To understand the wavelength dependence, it is important to note that a smaller dielectric constant and/or a larger overlap between electrons and holes lead to a greater binding energy. The decrease in the binding energy for longer wavelengths is due to the increased In composition because $\mathrm{InN}$ has a larger dielectric constant than GaN. For shorter wavelengths, the overlap between electrons and holes became smaller as discussed with Fig. 4(a), which weakens the exciton binding energy. This trend is more clearly observed for the 1-nm-thick QWs because the carrier confinement in 1-nm-thick QWs is weaker than that in 3-nm-thick QWs, which promotes carrier penetration into the barriers. The tilt dependence is caused by the difference in the electric field; stronger electric fields decrease the overlap between electrons and holes, and weaken the binding energy. 


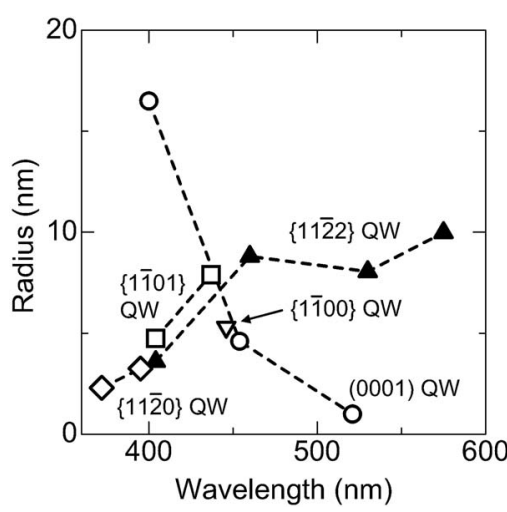

FIG. 7. Degree of localization estimated for the (0001), $\{11 \overline{2} 2\},\{1 \overline{1} 01\}$, $\{11 \overline{2} 0\}$, and $\{1 \overline{1} 00\}$ InGaN/GaN QWs.

The lateral confinement within a radius of $3 \mathrm{~nm}$ hardly affected the tendencies shown in Fig. 6 but enlarged the binding energy by $\sim 15 \mathrm{meV}$ for 1-nm-thick QDs and $\sim 13 \mathrm{meV}$ for 3-nm-thick QDs. This is due to the increased in-plane overlap between the electrons and holes. It is noteworthy that almost all the binding energies calculated for QDs exceeded the RT thermal energy of $26 \mathrm{meV}$, which was more remarkable in the semipolar and nonpolar QDs. Therefore, excitons in practical semipolar and nonpolar light emitters play an important role in determining the optical properties because $\mathrm{InGaN} / \mathrm{GaN}$ QWs generally involve lateral confinements due to naturally formed potential minima on the nanometer scale.

\section{DISCUSSION}

It has been reported that the polarization effect is difficult to be detected experimentally in wells thinner than $\sim 5 \mathrm{~nm}^{41}$ However, the present calculations indicate that both the polarization and localization effects can be detected even in 1-nm-thick quantum structures in terms of spontaneous emission lifetime. Here, we determined the degree of exciton localizations by comparing the experimentally obtained spontaneous emission lifetime shown in Fig. 1 to the calculations.

Because the well width was experimentally determined, it was treated as a constant in the current analysis. On the other hand, the In composition (and consequently electric field) and the radius for the lateral confinement $(R)$ were fitting parameters. Thus, Fig. 7 plots the obtained degrees of localizations, $R$, as a function of the emission wavelength. It can be seen that excitons in the semipolar QWs are more weakly localized in the longer wavelength range, and $R$ is nearly unchanged at $\sim 10 \mathrm{~nm}$ for wavelengths longer than $450 \mathrm{~nm}$. For the nonpolar QWs, the wavelength dependence is almost the same as that of the semipolar QWs. In contrast, excitons in the (0001) polar QWs are localized more strongly as the wavelength increases, which is consistent with previous reports (for example, Ref. 5). Although the reason for this remarkable difference between the polar QWs and semipolar and nonpolar QWs is unclear, it may be related to crystal growth. As noted in Ref. 44, adatoms on the $\{11 \overline{2} 2\}$ plane migrate more than those on the (0001) plane. Such crystal plane dependence can cause differing formation processes of the potential fluctuations and, consequently, cause different degrees of carrier localization.

\section{SUMMARY}

Excitonic properties of polar, semipolar, and nonpolar InGaN/GaN strained QWs were investigated. The spontaneous emission lifetimes measured at $\sim 10 \mathrm{~K}$ in the (0001) QWs ranged from $1.4 \mathrm{~ns}$ at $400 \mathrm{~nm}$ to $85 \mathrm{~ns}$ at $520 \mathrm{~nm}$, while those in the semipolar and nonpolar QWs remained on the order of a few hundred picoseconds. The theoretical calculation, which considered the exciton localization and polarization field, revealed that the size of the localization centers in the polar QWs was reduced for longer emission wavelengths, whereas those in the semipolar and nonpolar QWs showed the opposite tendency. The reason was attributed to the different growth characteristics. Furthermore, the calculated exciton binding energy in semipolar and nonpolar QDs was larger than the RT thermal energy, suggesting the importance of excitons in these QDs.

\section{ACKNOWLEDGMENTS}

The authors thank Dr. A. Kaneta and Dr. K. Nishizuka for providing valuable data. This work was partially supported by the Global COE Program and Grant-in-Aid for Scientific Research (A) by the Ministry of Education, Culture, Sports, Science and Technology.

${ }^{1}$ S. Nakamura, S. Pearton, and G. Fasol, Blue Laser Diode, 2nd ed. (Springer, Heidelberg, 2000).

${ }^{2}$ I.-h. Ho and G. B. Stringfellow, Appl. Phys. Lett. 69, 2701 (1996).

${ }^{3}$ S. Chichibu, T. Azuhata, T. Sota, and S. Nakamura, Appl. Phys. Lett. 69, 4188 (1996)

${ }^{4}$ Y. Narukawa, Y. Kawakami, M. Funato, Sz. Fujita, Sg. Fujita, and S. Nakamura, Appl. Phys. Lett. 70, 981 (1997).

${ }^{5}$ Y. Narukawa, Y. Kawakami, Sg. Fujita, and S. Nakamura, Phys. Rev. B 59, 10283 (1999).

${ }^{6}$ F. Bernardini and V. Fiorentini, Phys. Status Solidi B 216, 391 (1999).

${ }^{7}$ A. Hangleiter, J. S. Im, H. Kolmer, S. Heppel, J. Off, and F. Scholz, MRS Internet J. Nitride Semicond. Res. 3, 15 (1998).

${ }^{8}$ S.-H. Park and S.-L. Chung, Phys. Rev. B 59, 4725 (1999).

${ }^{9}$ T. Takeuchi, H. Amano, and I. Akasaki, Jpn. J. Appl. Phys., Part 1 39, 413 (2000).

${ }^{10}$ K. Nishizuka, M. Funato, Y. Kawakami, Sg. Fujita, Y. Narukawa, and T. Mukai, Appl. Phys. Lett. 85, 3122 (2004).

${ }^{11}$ K. Nishizuka, M. Funato, Y. Kawakami, Y. Narukawa, and T. Mukai, Appl. Phys. Lett. 87, 231901 (2005).

${ }^{12}$ M. Funato, T. Kotani, T. Kondou, Y. Kawakami, Y. Narukawa, and T. Mukai, Appl. Phys. Lett. 88, 261920 (2006).

${ }^{13}$ M. Ueda, K. Kojima, M. Funato, Y. Kawakami, Y. Narukawa, and T. Mukai, Appl. Phys. Lett. 89, 211907 (2006).

${ }^{14}$ M. Funato, M. Ueda, Y. Kawakami, T. Kosugi, M. Takahashi, Y. Narukawa, and T. Mukai, Jpn. J. Appl. Phys., Part 2 45, L659 (2006).

${ }^{15}$ M. Funato, S. Yamamoto, K. Kaisei, K. Shimogami, Sz. Fujita, and Sg. Fujita, Appl. Phys. Lett. 79, 4133 (2001).

${ }^{16}$ P. Waltereit, O. Brandt, A. Trampert, H. T. Grahn, J. Menniger, M. Ramsteiner, M. Reiche, and K. H. Ploog, Nature (London) 406, 865 (2000).

${ }^{17}$ A. Chitnis, C. Chen, V. Adivarahan, M. Shatalov, E. Kuokstis, V. Mandavilli, J. Yang, and M. A. Khan, Appl. Phys. Lett. 84, 3663 (2004).

${ }^{18}$ B. A. Haskell, F. Wu, S. Matsuda, M. D. Craven, P. T. Fini, S. P. DenBaars, J. S. Speck, and S. Nakamura, Appl. Phys. Lett. 83, 1554 (2003).

${ }^{19}$ H. M. Ng, A. Bell, F. A. Ponce, and S. N. G. Chu, Appl. Phys. Lett. 83, $653(2003)$.

${ }^{20}$ F. D. Sala, A. D. Carlo, P. Lugli, F. Bernardini, V. Fiorentini, R. Scholz, and J.-M. Jancu, Appl. Phys. Lett. 74, 2002 (1999).

${ }^{21}$ L.-H. Peng, Y.-C. Hsu, and C.-W. Chuang, IEEE J. Sel. Top. Quantum 
Electron. 5, 756 (1999).

${ }^{22}$ O. Mayrock, H.-J. Wünsche, and F. Henneberger, Phys. Rev. B 62, 16870 (2000).

${ }^{23}$ S. Kalliakos, P. Lefebvre, and T. Taliercio, Phys. Rev. B 67, 205307 (2003).

${ }^{24}$ T. Miyajima, Y. Kudo, K.-L. Liu, T. Uruga, H. Honma, Y. Saito, M. Hori, Y. Nanishi, T. Kobayashi, and S. Hirata, Phys. Status Solidi B 234, 801 (2002).

${ }^{25}$ K. Shimada, T. Sota, and K. Suzuki, J. Appl. Phys. 84, 4951 (1998).

${ }^{26}$ F. Bernardini, V. Fiorentini, and D. Vanderbilt, Phys. Rev. B 56, R10024 (1997).

${ }^{27}$ M. Yamaguchi, T. Yagi, T. Azuhata, T. Sota, K. Suzuki, S. Chichibu, and S. Nakamura, J. Phys.: Condens. Matter 9, 241 (1997).

${ }^{28}$ A. F. Wright, J. Appl. Phys. 82, 2833 (1997).

${ }^{29}$ A. S. Barker and M. Ilegems, Phys. Rev. B 7, 743 (1973).

${ }^{30}$ V. W. L. Chin, T. L. Tansley, and T. Osotchan, J. Appl. Phys. 75, 7365 (1994).

${ }^{31}$ A. Shikanai, T. Azuhata, T. Sota, S. Chichibu, A. Kuramata, K. Horino, and S. Nakamura, J. Appl. Phys. 81, 417 (1997).

${ }^{32}$ Y. C. Yeo, T. C. Chong, and M. F. Li, J. Appl. Phys. 83, 1429 (1998).

${ }^{33}$ J. Wu, W. Walukiewicz, K. M. Yu, J. W. Ager III, E. E. Haller, H. Lu, and W. J. Schaff, Phys. Status Solidi B 240, 412 (2003).
${ }^{34}$ Y. Nanishi, Y. Saito, and T. Yamaguchi, Jpn. J. Appl. Phys., Part 1 42, 2549 (2003).

${ }^{35}$ S.-H. Wei and A. Zunger, Appl. Phys. Lett. 69, 2719 (1996).

${ }^{36}$ S.-H. Park, J. Appl. Phys. 91, 9904 (2002).

${ }^{37}$ M. Sugawara, Phys. Rev. B 51, 10743 (1995)

${ }^{38}$ C. Gourdon and P. Lavallard, Phys. Status Solidi B 153, 641 (1989)

${ }^{39}$ A. Kaneta, K. Okamoto, Y. Kawakami, Sg. Fujita, G. Marutsuki, Y. Narukawa, and T. Mukai, Appl. Phys. Lett. 81, 4353 (2002).

${ }^{40}$ Spatial mapping acquired by the illumination-collection mode of SNOM revealed that the emission wavelength depends on the position, and fluctuates between 470 and $490 \mathrm{~nm}$. This inhomogeneity implies that the exciton localization is $\sim 100 \mathrm{meV}$ deep, which is consistent with the energy profile of the tail state derived from the weakly localization model, that is, $\exp (E / 35 \mathrm{meV})$.

${ }^{41}$ K. Omae, Y. Kawakami, Sg. Fujita, Y. Narukawa, and T. Mukai, Phys. Rev. B 68, 085303 (2003).

${ }^{42}$ S.-H. Park, D. Ahn, E. H. Park, T. K. Yoo, and Y.-T. Lee, Appl. Phys. Lett. 87, 044103 (2005).

${ }^{43}$ S.-H. Park and S.-L. Chuang, Appl. Phys. Lett. 76, 1981 (2000).

${ }^{44}$ M. Ueda, K. Hayashi, T. Kondou, M. Funato, Y. Kawakami, Y. Narukawa, and T. Mukai, Phys. Status Solidi C 4, 2826 (2007). 